\title{
Heart failure with preserved ejection fraction
}

\author{
Authors: Pierpaolo Pellicori ${ }^{\mathrm{A}}$ and John GF Cleland ${ }^{\mathrm{B}}$
}

\begin{abstract}
Many patients with heart failure (HF) have a normal left ventricular ejection fraction, and are labelled as having $\mathrm{HF}$ with preserved left ventricular ejection fraction (HFPEF). Hypertension, atrial fibrillation and age are important contributors to the development of HFPEF and, therefore, its prevalence is likely to increase in the next few decades. The pathophysiology of HFPEF is heterogeneous but with a final common pathway leading to congestion. HF remains a clinical diagnosis but the plasma concentration of B-type natriuretic peptide (eg BNP/N-terminal prohormone BNP (NT-proBNP)), a marker of congestion, is an essential component. Imaging, usually by echocardiography, is required to determine the cardiac phenotype (ie valve disease, left ventricular ejection fraction) underlying HF. A superficially normal echocardiogram does not exclude a diagnosis of HF. No treatment has been shown conclusively to alter the prognosis of HFPEF. However, treatments directed at congestion and hypertension, such as diuretics, mineralocorticoid receptor antagonists (MRAs) and angiotensin converting-enzyme inhibitors, may improve symptoms and probably do improve outcomes. No treatment has yet been shown to reverse the underlying myocardial pathology of HFPEF, although there is some hope that MRAs might.
\end{abstract}

KEYWORDS: Heart failure, preserved ejection fraction, morbidity, mortality, therapy

\section{Introduction}

Breathlessness on exertion is extremely common; it causes substantial disability and has many causes, including heart failure (HF). In England, HF causes or contributes to about 400,000 emergency admissions to hospital each year. About $10-15 \%$ of these patients will die in hospital and more than $20 \%$ of those surviving admission will die in the following year, many of them subsequent to a further admission. ${ }^{1}$

Many epidemiological reports suggest that about half of patients who have symptoms or signs of HF have a relatively normal left ventricular ejection fraction (LVEF) and are labelled as having HF with preserved ejection fraction (HFPEF). In the coming decades, as the population ages, the prevalence of

Authors: Aresearch fellow in clinical cardiology, Department of Cardiology, Hull York Medical School, Hull, UK; ${ }^{B}$ professor of clinical cardiology, Cardiovascular Biomedical Research Unit Royal Brompton and Harefield Hospitals, Imperial College, London, UK diabetes, obesity and hypertension increases and survival after myocardial infarction improves, the substrate for developing HF and its incidence will therefore increase dramatically., ${ }^{2,3}$

Over the last 25 years, several effective therapies have been identified for patients who have HF with reduced ejection fraction (HFREF) but none have been conclusively shown to be effective for HFPEF. This may reflect problems in either the veracity of the diagnosis of HF or the heterogeneous pathophysiology of HFPEF that renders it unamenable to a single therapeutic approach.

\section{What is HFPEF?}

Many attribute HFPEF predominantly to left ventricular diastolic dysfunction (DD). However, many patients with DD have no readily identifiable symptoms. They may become breathless during exercise but this is often attributed to age and low levels of fitness. They are probably more prone to developing clinically overt HFPEF but DD is not an accurate predictor of risk. ${ }^{4}$ The underlying myocardial pathology appears to be heterogeneous but many patients have left ventricular and cardiac myocyte hypertrophy with impaired relaxation and an increase in myocardial collagen content indicating fibrosis. Opinions differ on which is most important in HFPEF; most likely this varies among patients. In some patients, scars indicating subclinical myocardial infarction can be found. Amyloid may be an under-recognised problem, especially senile or transthyretin cardiac amyloidosis, which run a more benign course than light-chain induced amyloidosis. ${ }^{5}$

Breathlessness on exercise is the key clinical marker of HFPEF. Patients with HFPEF and controls often have a similar haemodynamic profile at rest. The haemodynamic hallmark of HFPEF is an exaggerated rise in pulmonary capillary wedge pressure and pulmonary artery pressure during exercise. ${ }^{6}$ This is accompanied by a failure of $\mathrm{LV}$ end-diastolic volume to increase during exercise (impaired diastolic reserve) due to impaired left ventricular relaxation and increased left ventricular stiffness, ${ }^{7,8}$ leading to an attenuated increase in stroke volume and cardiac output. Exercise-induced mitral regurgitation and left ventricular dyssynchrony may also contribute to abnormal haemodynamics and impaired exercise capacity. ${ }^{9}$ However, skeletal muscle deconditioning and respiratory and joint disease most likely also have an important impact on exercise capacity.

HFPEF-associated morphological changes occurring in the heart structure include focal (scar, due to myocardial infarction) or diffuse (due to hypertension or inflammation) fibrosis, leading initially to a decline in long-axis LV systolic and diastolic function. ${ }^{10}$ Systolic and diastolic segmental impairment of LV function, such as a reduced radial function and apical rotation, 
Hypertension

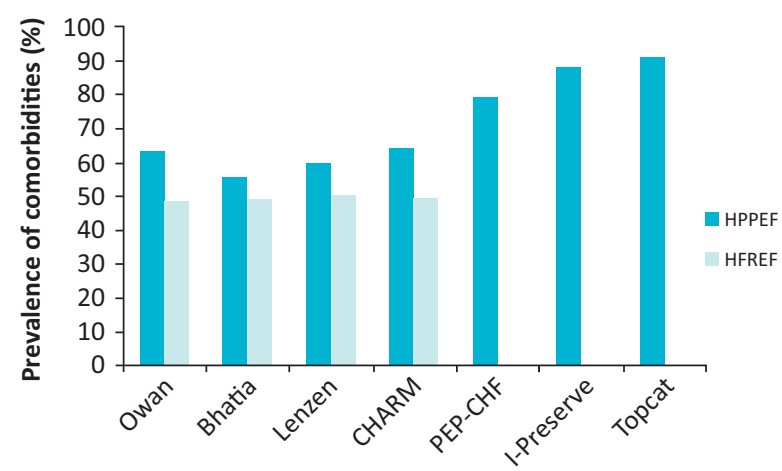

Atrial fibrillation

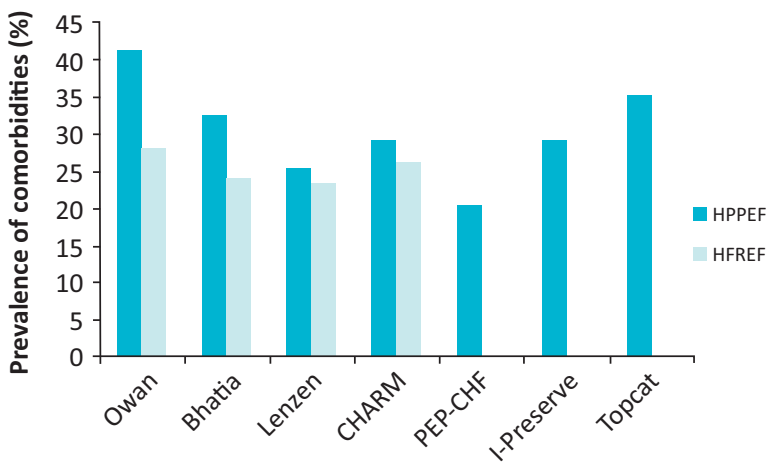

Ischaemic heart disease

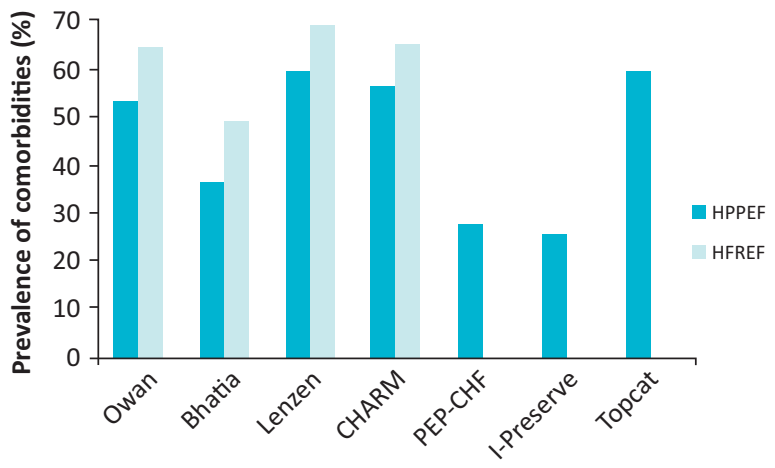

Diabetes

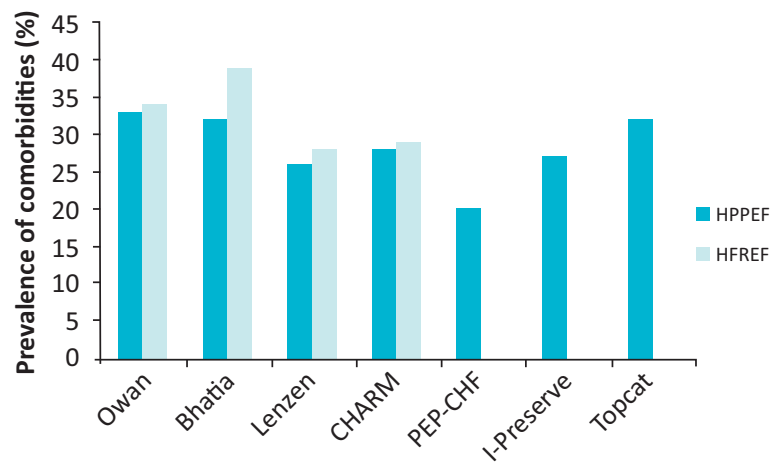

Fig 1. Prevalence of important comorbidities (atrial fibrillation, hypertension, ischaemic heart disease and diabetes) among patients with HFPEF compared with those with HFREF in observational studies and relevant clinical trials. ${ }^{12-17}$ HFPEF = heart failure with preserved left ventricular ejection fraction; HFREF = HF with reduced ejection fraction.

or delayed LV untwisting, can also be detected with advanced echocardiographic methods. ${ }^{11}$

However, HFPEF is a disease of the elderly and it is not surprising that several comorbidities have a higher prevalence among patients with HFPEF than in those with HFREF (Fig 1), including atrial fibrillation, anaemia, chronic obstructive pulmonary disease (COPD), renal dysfunction and, most notably, hypertension. Many of these will actively contribute to the development of HFPEF and are not merely 'bystanders'. Arterial stiffening is another age-related process that is both a cause and consequence of hypertension that may increase LV afterload at rest, and which may be exaggerated during exercise. ${ }^{18}$
Thus, from a narrow cardiology perspective, HFPEF can be considered a clinical condition characterised by both systolic and diastolic left ventricular dysfunction. However, from a broader clinical perspective, HFPEF might be considered an age-related syndrome with LV dysfunction as only one component of a multi-system disorder that contributes to symptoms (breathlessness) and signs (peripheral oedema). ${ }^{19}$

Reports from registries suggest that the outcome of patients with HFPEF and HFREF might be similar, ${ }^{12}$ but clinical trials (that usually exclude patients with comorbidities that cause diagnostic confusion) have not confirmed this (Fig 2). The poor prognosis of HFPEF may reflect the concomitant effects
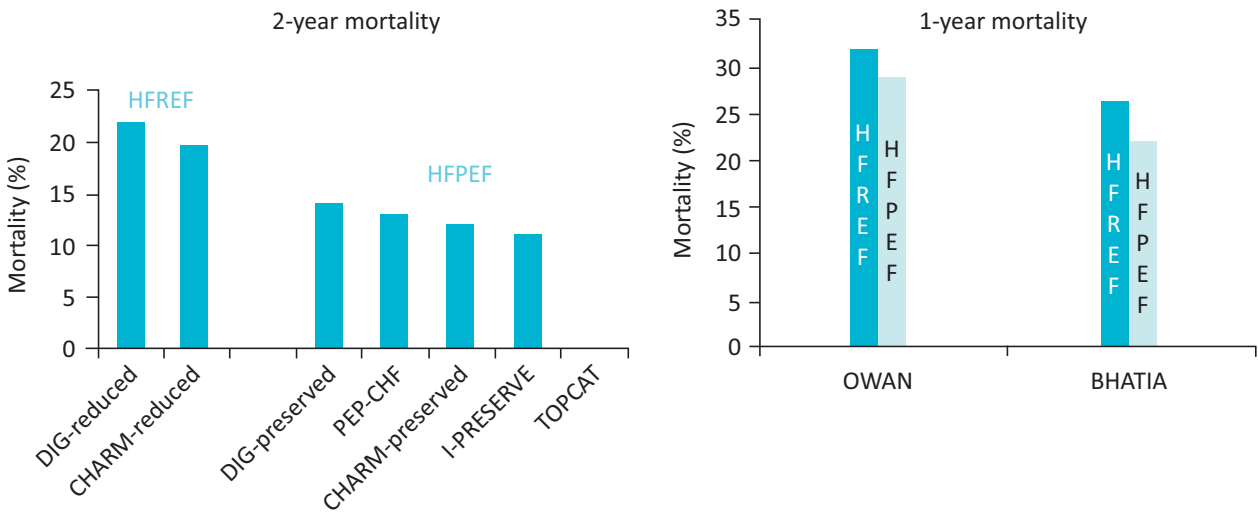

Fig 2. Mortality in trials (left) and observational studies (right) in HFPEF and HRFEF., 32 HFPEF $=$ heart failure with preserved left ventricular ejection fraction; HFREF = heart failure with reduced ejection fraction. 
of greater age and the number of other serious comorbidities, rather than the severity of cardiac dysfunction.

\section{How is HFPEF diagnosed?}

The diagnosis of HFPEF is probably often overlooked. Only about one-third of patients treated with loop diuretics are labelled as HF, but when investigated many are found to have cardiac dysfunction. The diagnosis of HFPEF may also often be made in error. ${ }^{9}$ However it is also likely that HFPEF is a paroxysmal disease. Although LVDD may be consistently present, the evidence for HF may only appear when the heart is stressed by, for example, uncontrolled hypertension, atrial fibrillation, anaemia or infection..$^{19}$ This explains why many patients admitted with florid features of HF have little or no evidence of the disease at subsequent outpatient follow-up. In a breathless patient, a diagnosis of HF can be made with some confidence when the LVEF is reduced, even when comorbid respiratory disease exists. When LVEF is normal, there are two approaches to the diagnosis of HFPEF: either exclusion of alternative reasons for symptoms and signs and the use of clinical judgment or a diagnosis based on evidence of cardiac dysfunction other than a reduced LVEF. Unfortunately, as noted above, many older patients have abnormal DD but no symptoms.

The 2007 European Society of Cardiology consensus statement required echocardiographic $\left(\mathrm{E}^{\mathrm{E}} \mathrm{E}^{\prime}>15\right)$ or invasive (raised left atrial or left ventricular filling pressure) evidence of diastolic dysfunction to confirm a diagnosis of HFPEF when LVEF $>50 \% .{ }^{20}$ It is worth mentioning that these authors were the first to introduce the use of natriuretic peptides for diagnostic purposes, but only when supported by echocardiographic measurements (E/E'>8). However, the guidance on natriuretic peptides was flawed for many reasons. Suggested diagnostic thresholds for B-type natriuretic peptide (BNP) (>200 pg/ml) and N-terminal prohormone BNP (NT-pro BNP); >220 pg/ml/l) were inconsistent and no account was taken of heart rhythm or renal function, both of which have a profound effect on the interpretation of results. The recent European Society of Heart Failure (ESC) HF guidelines lowered the cut-off for NTproBNP plasma levels $(<125 \mathrm{ng} / \mathrm{l})$ to exclude serious cardiac structural alterations or dysfunction, but the diagnosis of HFPEF still requires identification of diastolic dysfunction by echocardiography. ${ }^{21}$ Importantly, this set of guidelines suggested that a single echocardiographic variable should not be used to establish a diagnosis, but a more comprehensive assessment of LV structure (volumes and mass), left atrial size and Doppler variables is required. The American Heart Association and the American College of Cardiology (ACC/AHA) guidelines for the management of HF have introduced an important distinction between those patients who have HF and relatively preserved LVEF (>40\%), with three groups of patients being identified: 'possible' HFPEF (LVEF $>50 \%$ ), 'borderline' HFPEF $(40<$ LVEF $<49)$, and 'improved' HFPEF (LVEF $>40 \%)$, for patients who had HFREF whose LVEF had risen but in whom the features of HF had not resolved. ${ }^{22}$

Although there is still debate about whether HFPEF and HFREF are a continuum of the same disease or two separate clinical entities, ${ }^{23,24}$ differences exist in pathophysiology, the importance of hypertension and ischaemic heart disease to

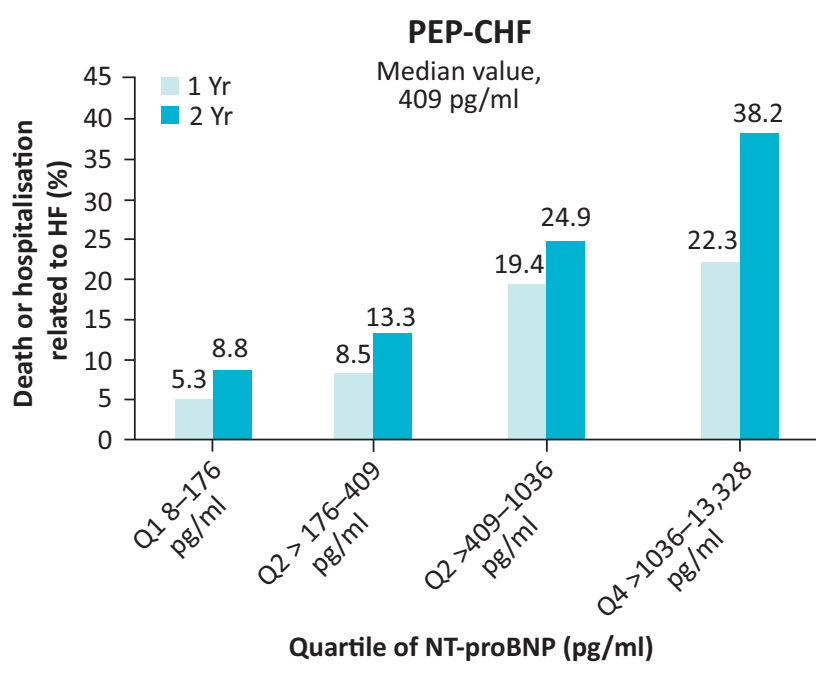

Fig 3. Outcome according to quartiles of the plasma concentration of NT-proBNP in patients with HFPEF enrolled in the PEP-CHF study. Reproduced with permission from Cleland et al.15 $\mathrm{HF}=$ heart failure; $\mathrm{HFPEF}=$ heart failure with preserved left ventricular ejection fraction; NT-proBNP = $\mathrm{N}$-terminal prohormone B-type natriuretic peptide.

causation and in treatment. ${ }^{25}$ Therefore, it is important to identify cardiac phenotypes that are hidden behind the same acronym and a single echocardiographic measurement.

Natriuretic peptides are produced by the heart as a defence against fluid congestion. They are an essential tool to identify both patients with HF and their risk of clinical events (Fig 3). ${ }^{15,26}$ However, natriuretic peptides are almost completely agnostic as to the cause of congestion, including cardiac or renal causes. The rare exception is constrictive pericarditis, where severe congestion may develop with only a modest increase in natriuretic peptides, the plasma concentration of which reflects transmural wall stress rather than intra-cardiac pressure. ${ }^{27}$ When natriuretic peptide levels are low, clinicians can generally reassure themselves and their patients that breathlessness or oedema are not cardiac in origin and that prognosis is good. ${ }^{28}$ Increased plasma concentrations of natriuretic peptides suggest that the heart is stressed and congestion is developing. Plasma concentrations do rise with age, renal dysfunction, the severity of coronary disease, anaemia and heart rhythm disturbances, such as atrial fibrillation, because each of these places a strain on the heart. Each also brings the patient closer to death. ${ }^{29}$ Only those interested in rationing care to older people should adjust interpretation of plasma concentrations of natriuretic peptides for age. ${ }^{30}$

Taking the above to its logical but provocative conclusion, the diagnosis of HF should be based on clinical assessment made in the light of the plasma concentration of BNP/NT-proBNP ${ }^{31}$ and not be based on echocardiography. However, imaging plays an essential role in identifying the cardiac phenotype and probable cause of HF by assessing LV contractile function, left atrial volume and valve function. A broader echocardiographic perspective that focuses on the up-stream consequences of a stiffer left ventricle might be the best method to phenotype this complex disease further and to identify different therapeutic targets. ${ }^{32}$ Other data suggest that assessing RV function or even the vena cava may be a 

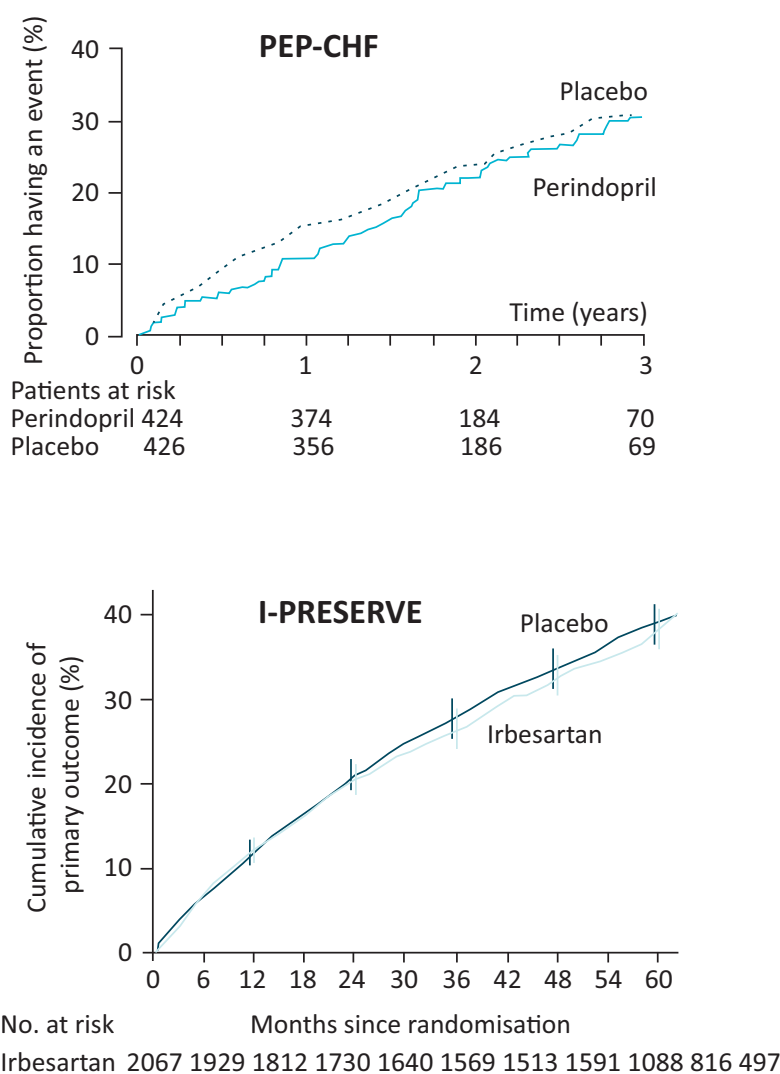

Placebo 206119211808171516181539146612461051776446
No. at risk Candesartan Placebo
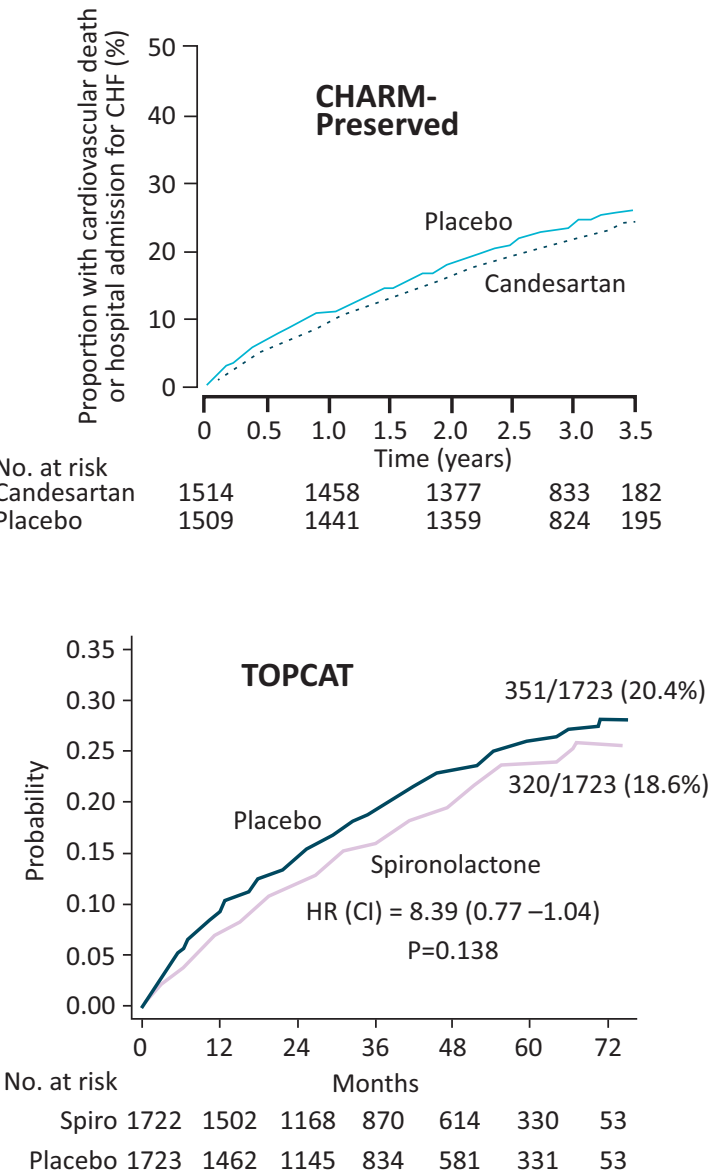

Fig 4. Primary outcomes of large, contemporary randomised controlled trials of HFPEF. ${ }^{14-17}$ Primary outcome definitions: CHARM-Preserved: cardiovascular death or admission to hospital for chronic HF; PEP-CHF: composite of all-cause mortality and unplanned HF related hospitalisation; I-PRESERVE: death from any cause or hospitalisation for a cardiovascular cause (HF, myocardial infarction, unstable angina, arrhythmia or stroke); TOPCAT: death due to cardiovascular causes, aborted cardiac arrest, or hospitalisation for the management of HF. CI = confidence interval; HF = heart failure; HFPEF = heart failure with preserved left ventricular ejection fraction; $\mathrm{HR}=$ hazard ratio. Images reproduced with permission. ${ }^{14-17}$

better guide to prognosis than measures of left heart function, but these may be late findings that are of less value for diagnosis. ${ }^{33}$

\section{How should HFPEF be managed?}

Current ESC guidelines ${ }^{21}$ highlight that there is, so far, no robust evidence that any treatment can reduce morbidity and mortality in patients with HFPEF (Fig 4).

Diuretics (either loop diuretics or thiazides) offer an effective treatment for congestion and the rapid relief of symptoms that may also improve quality of life. ${ }^{34}$ There are fears that conventional diuretics may accelerate the progression of cardiovascular dysfunction by activating the renin-angiotensin-aldosterone system. There is a powerful body of evidence that thiazide diuretics reduce morbidity and mortality among older patients with hypertension, ${ }^{35,36}$ many of whom probably had or would soon develop HFPEF. There is no such evidence for loop diuretics.

Calcium-channel blockers (CCB), a class of drug contraindicated in patients with HFREF, improved exercise tolerance and LV diastolic function in small studies of highly selected patients with HFPEF, as shown by two small crossover studies conducted in the 1990s that used verapamil. ${ }^{37,38}$ Calcium channel blockers might also be used for ventricular rate control for patients in atrial fibrillation, although the optimal target heart rate is unknown. ${ }^{39} \mathrm{~A}$ recent trial compared two CCB (verapamil and diltiazem) to two beta blockers (BB) (metoprolol and carvedilol) in a randomised cross-over study that enrolled 60 patients with permanent atrial fibrillation and grossly elevated plasma concentrations of NT-proBNP (mean 1039 [SD 636] ng/l) that could not be attributed solely to atrial fibrillation, although none of the patients had overt HF. The rate of adverse events leading to discontinuation of medication was relatively high (25\%), mainly related to the use of $\mathrm{BB}$ (10 patients reported fatigue and dyspnoea while taking $\mathrm{BB}$, versus only two on CCB). Treatment with CCB, but not with BB, preserved exercise capacity and was associated with a decline in NT-proBNP compared with baseline. ${ }^{40}$

It is likely, although the evidence is not conclusive, that patients with HFPEF benefit from treatment with angiotensinconverting-enzyme (ACE) inhibitors. Hypertension commonly causes or contributes to the development of HFPEF and lowering blood pressure might be expected to delay the onset or progression of HF. 
Table 1. Primary outcome in TOPCAT for patients enrolled because they had a previous HF-related admission in the previous 12 months or an elevated plasma concentration of BNP (>100 pg/ml) or NT-proBNP (>360 pg/ml).

\begin{tabular}{|c|c|c|c|c|c|}
\hline Outcome & Spironolactone (\%) & \%/year & Placebo (\%) & \%/year & $\mathrm{OR}(\mathrm{CI})$ \\
\hline \multicolumn{6}{|c|}{ Entry criterion - prior hospitalisation } \\
\hline Primary & $242(19.6)$ & 6.0 & $235(19.1)$ & 6.0 & $1.01(0.84-1.21)$ \\
\hline CV death & $120(9.7)$ & 2.8 & $117(9.5)$ & 2.8 & $1.01(0.78-1.31)$ \\
\hline HF hospitalisation & $151(12.3)$ & 3.8 & $162(13.1)$ & 4.1 & $0.92(0.73-1.14)$ \\
\hline \multicolumn{6}{|c|}{ Entry criterion - natriuretic peptides } \\
\hline Primary & 78 (15.9) & 5.5 & $116(23.6)$ & 8.5 & $\begin{array}{l}0.65(0.49-0.87) \\
p=0.003\end{array}$ \\
\hline CV death & $40(8.2)$ & 2.7 & 59 (12.0) & 3.9 & $\begin{array}{l}0.69(0.46-1.03) \\
p=0.069\end{array}$ \\
\hline HF hospitalisation & $55(11.2)$ & 3.9 & $83(16.9)$ & 6.1 & $\begin{array}{l}0.64(0.46-0.90) \\
p=0.011\end{array}$ \\
\hline
\end{tabular}

The primary outcome was death due to cardiovascular causes, aborted cardiac arrest, or hospitalisation for the management of HF. Patients enrolled because of a prior hospital admission for HF had fewer events and did not benefit from spironolactone. Those enrolled due to an increase in natriuretic peptides, indicating congestion, had a higher rate of events and obtained substantial benefit from spironolactone that was similar in magnitude to that observed for HFREF. CI = confidence interval; $\mathrm{CV}=$ cardiovascular; $\mathrm{HF}=$ heart failure; HFREF = HF with reduced ejection fraction; NT-proBNP = N-terminal prohormone B-type natriuretic peptide; OR = odds ratio.

In the HYVET study, ${ }^{36} 3,845$ elderly patients (mean age of 84 years, $60 \%$ female) were randomised to indapamide (with or without perindopril) or placebo: a significant reduction in the development of HF and in mortality was observed after a follow-up period of 1.8 years. In the PEP-CHF trial, perindopril improved symptoms and exercise capacity at one year and this was associated with a reduction in the rate of composite allcause mortality and HF hospitalisations (the primary endpoint) that were similar in magnitude to that observed with enalapril in patients with HFREF. ${ }^{15,41}$ The evidence of benefit for angiotensin II receptor blockers is weaker, ${ }^{14,16}$ just as it is for HFREF, although, curiously, a subgroup analysis of the I-PRESERVE trial suggests that patients with lower plasma concentrations of natriuretic peptides might derive some outcome benefits. ${ }^{29}$

Several smaller and two substantial studies of mineralocorticoid receptor antagonists (MRA) have suggested equivocal benefits for patients with HFPEF. ${ }^{17,28,42}$ The population enrolled in ALDO-DHF had rather mild diastolic dysfunction with little evidence of $\mathrm{HF}$ and a good prognosis. In TOPCAT, patients with an elevated plasma concentration of natriuretic peptides, indicating congestion and confirming that they had HFPEF, had a reduction in morbidity and mortality with the addition of spironolactone compared with placebo (Table 1). The magnitude of benefit was similar to that observed in HFREF. Patients who were enrolled based on clinical features alone had few events and did not benefit from spironolactone.

No randomised controlled trial (RCT) has been designed to evaluate specifically the role of BB in HFPEF. SENIORS enrolled more than 2,000 patients with HF, and one-third had LVEF $>35 \%$. In a pre-specified sub-analysis, nebivolol exerted a similar $20 \%$ reduction in the primary end point (all-cause mortality or cardiovascular hospitalisations) among patients with either HFREF or HFPEF, although the trial was not powered to show a statistically significant effect of nebivolol in different LVEF subgroups. ${ }^{43}$ Although higher heart rate is associated with an adverse outcome in both HFREF and HFPEF, there is very limited evidence that ivabradine is effective in improving either function or outcome. A moderately large study is underway.

Digoxin is an old drug that should receive more attention. In a randomised trial of 988 patients with clinical HFPEF in sinus rhythm, digoxin reduced the primary composite endpoint of hospitalisation or death due to HF at two years but, disappointingly, the effect was no longer apparent by the end of the study at 37 months. ${ }^{44}$

New treatments being developed for various forms of cardiac amyloidosis will require greater diagnostic efforts to identify appropriate patients.

\section{Devices}

Left ventricular dyssynchrony might contribute to the onset of symptoms in HFPEF, particularly when the QRS interval is prolonged. Although isolated case reports and retrospective analysis of previous studies suggest that some patients with LVEF $>40 \%$ might benefit from cardiac resynchronisation therapy, no RCT have been reported. ${ }^{45,46}$ There is no evidence that patients with HFPEF benefit from defibrillators. Implanted monitoring devices might help guide treatment and could be a major advance in management, but their outcome impact is still unknown. ${ }^{47}$ Similarly, inter-atrial shunt devices, designed to off-load left atrial pressure into the systemic venous circulation in a controlled fashion to prevent the exaggerated rise in left atrial pressure during exercise, could be a novel way to manage patients recalcitrant to conventional management of symptoms. ${ }^{48}$

\section{Conclusions}

HFPEF predominantly affects older patients and is characterised by increased left atrial pressure, especially during 
exercise, in the absence of LV dilatation or markedly depressed LVEF. Natriuretic peptides are the key means of detecting increases in atrial pressure due to congestion. Education and experience is required to interpret plasma concentrations effectively. Imaging reveals diverse and heterogeneous cardiac phenotypes underlying HFPEF that, in turn, may reflect diverse myocardial pathologies including hypertrophy, delayed cardiac myocyte relaxation, myocardial fibrosis and senile amyloidosis. Treatments directed at congestion (and/or hypertension), such as diuretics MRA and ACE inhibitors, may be similarly effective in HFPEF and HFREF. Treatments directed at myocardial pathology have so far failed to change the natural history of HFPEF as they have done for HFREF.

\section{References}

1 Cleland JG, McDonagh T, Rigby AS et al. National Heart Failure Audit Team for England and Wales. The national heart failure audit for England and Wales 2008-2009. Heart 2011;97:876-86.

2 Chen HH, Lainchbury JG, Senni M et al. Diastolic heart failure in the community: clinical profile, natural history, therapy, and impact of proposed diagnostic criteria. J Card Fail 2002;8:279-87.

3 Owan TE, Hodge DO, Herges RM et al. Trends in prevalence and outcome of heart failure with preserved ejection fraction. $\mathrm{N} \mathrm{Engl} \mathrm{J}$ Med 2006;355:251-9.

4 Zile MR, Gottdiener JS, Hetzel SJ et al. I-PRESERVE Investigators. Prevalence and significance of alterations in cardiac structure and function in patients with heart failure and a preserved ejection fraction. Circulation 2011;124:2491-501.

5 Mohammed SF, Mirzoyev SA, Edwards WD et al. Left ventricular amyloid deposition in patients with heart failure and preserved ejection fraction. JACC Heart Fail 2014;2:113-22.

6 Borlaug BA, Nishimura RA, Sorajja P et al. Exercise hemodynamics enhance diagnosis of early heart failure with preserved ejection fraction. Circ Heart Fail 2010;3:588-95.

7 Borlaug BA, Jaber WA, Ommen SR et al. Diastolic relaxation and compliance reserve during dynamic exercise in heart failure with preserved ejection fraction. Heart 2011; 97:964-9.

8 Zile MR, Baicu CF, Gaasch WH. Diastolic heart failure - abnormalities in active relaxation and passive stiffness of the left ventricle. $\mathrm{N} \mathrm{Engl} \mathrm{J}$ Med 2004;350:1953-9.

9 Penicka M, Bartunek J, Trakalova $\mathrm{H}$ et al. Heart failure with preserved ejection fraction in outpatients with unexplained dyspnea: a pressure-volume loop analysis. J Am Coll Cardiol 2010;55:1701-10.

10 Nikitin NP, Loh PH, de Silva R et al. Left ventricular morphology, global and longitudinal function in normal older individuals: a cardiac magnetic resonance study. Int J Cardiol 2006;108:76-83.

11 Tan YT, Wenzelburger F, Lee E et al. The pathophysiology of heart failure with normal ejection fraction: exercise echocardiography reveals complex abnormalities of both systolic and diastolic ventricular function involving torsion, untwist, and longitudinal motion. J Am Coll Cardiol 2009;54:36-46.

12 Bhatia RS, Tu JV, Lee DS et al. Outcome of heart failure with preserved ejection fraction in a population-based study. $\mathrm{N} \mathrm{Engl} \mathrm{J}$ Med 2006;355:260-9.

13 Lenzen MJ, Scholte op Reimer WJ, Boersma E et al. Differences between patients with a preserved and a depressed left ventricular function: a report from the EuroHeart Failure Survey. Eur Heart $J$ 2004;25:1214-20

14 Yusuf S, Pfeffer MA, Swedberg K et al. CHARM Investigators and Committees. Effects of candesartan in patients with chronic heart failure and preserved left-ventricular ejection fraction: the CHARM-Preserved Trial. Lancet 2003;362:777-81.
15 Cleland JG, Taylor J, Freemantle N et al. Relationship between plasma concentrations of $\mathrm{N}$-terminal pro brain natriuretic peptide and the characteristics and outcome of patients with a clinical diagnosis of diastolic heart failure: a report from the PEP-CHF study. Eur J Heart Fail 2012;14:487-94.

16 Massie BM, Carson PE, McMurray JJ et al. I-PRESERVE Investigators. Irbesartan in patients with heart failure and preserved ejection fraction. N Engl J Med 2008;359:2456-67.

17 Pitt B, Pfeffer MA, Assmann SF et al. TOPCAT Investigators. Spironolactone for heart failure with preserved ejection fraction. N Engl J Med 2014;370:1383-92.

18 Chen CH, Nakayama M, Nevo E et al. Coupled systolic-ventricular and vascular stiffening with age: implications for pressure regulation and cardiac reserve in the elderly. J Am Coll Cardiol 1998;32:1221-7.

19 Banerjee P, Clark AL, Nikitin N, Cleland JG. Diastolic heart failure. Paroxysmal or chronic? Eur J Heart Fail 2004;6:427-31.

20 Paulus WJ, Tschöpe C, Sanderson JE et al. How to diagnose diastolic heart failure: a consensus statement on the diagnosis of heart failure with normal left ventricular ejection fraction by the Heart Failure and Echocardiography Associations of the European Society of Cardiology. Eur Heart J 2007; 28:2539-50.

21 McMurray JJ, Adamopoulos S, Anker SD et al. ESC guidelines for the diagnosis and treatment of acute and chronic heart failure 2012: The Task Force for the Diagnosis and Treatment of Acute and Chronic Heart Failure 2012 of the European Society of Cardiology. Developed in collaboration with the Heart Failure Association (HFA) of the ESC. Eur J Heart Fail 2012;14:803-69.

22 Yancy CW, Jessup M, Bozkurt B et al. 2013 ACCF/AHA Guideline for the Management of Heart Failure: Executive Summary: A Report of the American College of Cardiology Foundation/ American Heart Association Task Force on Practice Guidelines. Circulation 2013;128:1810-52.

23 Borlaug BA, Redfield MM. Diastolic and systolic heart failure are distinct phenotypes within the heart failure spectrum. Circulation 2011;123:2006-13

24 De Keulenaer GW, Brutsaert DL. Systolic and diastolic heart failure are overlapping phenotypes within the heart failure spectrum. Circulation 2011;123:1996-2004; discussion 2005.

25 Brouwers FP, de Boer RA, van der Harst P et al. Incidence and epidemiology of new onset heart failure with preserved vs. reduced ejection fraction in a community-based cohort: 11-year follow-up of PREVEND. Eur Heart J 2013;34:1424-31.

26 Pellicori P, Kallvikbacka-Bennett A, Khaleva O et al. Global longitudinal strain in patients with suspected heart failure and a normal ejection fraction: does it improve diagnosis and risk stratification? Int J Cardiovasc Imaging 2014;30:69-79.

27 Karaahmet T, Yilmaz F, Tigen $\mathrm{K}$ et al. Diagnostic utility of plasma $\mathrm{N}$-terminal pro-B-type natriuretic peptide and C-reactive protein levels in differential diagnosis of pericardial constriction and restrictive cardiomyopathy. Congest Heart Fail 2009;15:265-70.

28 Cleland JG, Pellicori P. Defining diastolic heart failure and identifying effective therapies. JAMA 2013;309:825-6.

29 Anand IS, Rector TS, Cleland JG et al. Prognostic value of baseline plasma amino-terminal pro-brain natriuretic peptide and its interactions with irbesartan treatment effects in patients with heart failure and preserved ejection fraction: findings from the I-PRESERVE trial. Circ Heart Fail 2011;4:569-77.

30 Frankenstein L, Clark AL, Goode K et al. The prognostic value of individual NT-proBNP values in chronic heart failure does not change with advancing age. Heart 2009;95:825-9.

31 Pellicori P, Hutchinson K, Clark AL, Cleland JGF. Does speckle tracking really improve diagnosis and risk stratification in patients with HeFNEF? J Am Coll Cardiol 2014;64:1535. 
32 Pellicori P, Kallvikbacka-Bennett A, Zhang J et al. Revisiting a classical clinical sign: jugular venous ultrasound. Int J Cardiol 2014;170:364-70.

33 Pellicori P, Carubelli V, Zhang J et al. IVC diameter in patients with chronic heart failure: relationships and prognostic significance. JACC Cardiovasc Imaging 2013;6:16-28.

34 Yip GW, Wang M, Wang T et al. The Hong Kong diastolic heart failure study: a randomised controlled trial of diuretics, irbesartan and ramipril on quality of life, exercise capacity, left ventricular global and regional function in heart failure with a normal ejection fraction. Heart 2008;94:573-80.

35 SHEP Cooperative Research Group. Prevention of stroke by antihypertensive drug treatment in older persons with isolated systolic hypertension. Final results of the Systolic Hypertension in the Elderly Program (SHEP). JAMA 1991;265:3255-64.

36 Beckett NS, Peters R, Fletcher AE et al; HYVET Study Group. Treatment of hypertension in patients 80 years of age or older. N Engl J Med 2008; 358:1887-98.

37 Setaro JF, Zaret BL, Schulman DS et al. Usefulness of verapamil for congestive heart failure associated with abnormal left ventricular diastolic filling and normal left ventricular systolic performance. Am J Cardiol 1990;66:981-6.

38 Hung MJ, Cherng WJ, Kuo LT, Wang CH. Effect of verapamil in elderly patients with left ventricular diastolic dysfunction as a cause of congestive heart failure. Int J Clin Pract 2002;56:57-62.

39 Cullington D, Goode KM, Zhang J et al. Is heart rate important for patients with heart failure in atrial fibrillation? JACC Heart Fail 2014;2:213-20.

40 Ulimoen SR, Enger S, Pripp AH et al. Calcium channel blockers improve exercise capacity and reduce N-terminal Pro-B-type natriuretic peptide levels compared with beta-blockers in patients with permanent atrial fibrillation. Eur Heart J 2014;35:517-24.

41 The SOLVD Investigators. Effect of enalapril on survival in patients with reduced left ventricular ejection fractions and congestive heart failure. N Engl J Med 1991;325:293-302.
42 Edelmann F, Wachter R, Schmidt AG et al. Aldo-DHF Investigators. Effect of spironolactone on diastolic function and exercise capacity in patients with heart failure with preserved ejection fraction: the Aldo-DHF randomized controlled trial. JAMA 2013;309:781-91.

43 van Veldhuisen DJ, Cohen-Solal A, Böhm M et al. SENIORS Investigators. Beta-blockade with nebivolol in elderly heart failure patients with impaired and preserved left ventricular ejection fraction: Data From SENIORS (Study of Effects of Nebivolol Intervention on Outcomes and Rehospitalization in Seniors With Heart Failure). J Am Coll Cardiol 2009;53:2150-8.

44 Ahmed A, Rich MW, Fleg JL et al. Effects of digoxin on morbidity and mortality in diastolic heart failure: the ancillary digitalis investigation group trial. Circulation 2006;114:397-403.

45 Penicka M, Kocka V, Herman D et al. Cardiac resynchronization therapy for the causal treatment of heart failure with preserved ejection fraction: insight from a pressure-volume loop analysis. Eur J Heart Fail 2010;12:634-6.

46 Chung ES, Katra RP, Ghio $S$ et al. Cardiac resynchronization therapy may benefit patients with left ventricular ejection fraction >35\%: a PROSPECT trial substudy. Eur J Heart Fail 2010;12:581-7.

47 Hutchinson K, Pellicori P, Dierckx R et al. Remote telemonitoring for patients with heart failure: might monitoring pulmonary artery pressure become routine? Expert Rev Cardiovasc Ther 2014;12:1025-33.

48 Søndergaard L, Reddy V, Kaye D et al. Transcatheter treatment of heart failure with preserved or mildly reduced ejection fraction using a novel interatrial implant to lower left atrial pressure. Eur J Heart Fail 2014;16:796-801.

Address for correspondence: Dr P Pellicori, Department of Cardiology, Hull York Medical School, Hull and East Yorkshire Medical Research and Teaching Centre, Castle Hill Hospital, Cottingham, Kingston upon Hull, HU16 5JQ, UK.

Email: pierpaolo.pellicori@hey.nhs.uk 